Revue internationale P.M.E.

Économie et gestion de la petite et moyenne entreprise

\title{
Ressources ou barrières à l'exportation ? L'impact des réseaux locaux pour les PME nordiques
}

\section{Heikki Eskelinen et Eirik Vatne}

Volume 9, numéro 3-4, 1996

URI : https://id.erudit.org/iderudit/1008268ar

DOI : https://doi.org/10.7202/1008268ar

Aller au sommaire du numéro

Éditeur(s)

Presses de l’Université du Québec

ISSN

0776-5436 (imprimé)

1918-9699 (numérique)

Découvrir la revue

Citer cet article

Eskelinen, H. \& Vatne, E. (1996). Ressources ou barrières à l'exportation ? L'impact des réseaux locaux pour les PME nordiques. Revue internationale P.M.E., 9(3-4), 67-94. https://doi.org/10.7202/1008268ar
Résumé de l'article

Cette étude comparative de /'internationalisation des petites et moyennes entreprises est basée sur une enquête dans quatre régions types de trois pays scandinaves (Finlande, Norvège et Suède), soit 12 régions. L'objectif est de mesurer la signification du milieu local de l'entreprise et l'étendue des relations interfirmes sur le niveau d'internationalisation. Les entreprises sont groupées en trois catégories : exportatrices engagées, exportatrices sporadiques et non exportatrices. En termes de relations verticales et horizontales de coopération, les exportateurs engagés ne se distinguent guère des autres catégories. Les différences entre les régions types et entre les pays sont peu importantes quand il s'agit de l'utilisation des ressources internes dans les entreprises. Les différences dans le recours aux ressources externes sont toutefois plus importantes entre les pays qu'entre les régions. Les meilleurs indicateurs de succès des entreprises comme exportatrices sont le recours aux technologies de production et les types de produits offerts. 


\title{
Ressources ou barrières à l'exportation? L'impact des réseaux locaux pour les PME nordiques
}

Heikki ESKELINEN

Institut Karelien, Université de Joensuu

Eirik VATNE

SNF, Norwegian School of Economics and Business Administration

\section{PME - Internationalisation - Comportement d'exportation Relations interfirmes - Réseaux - Ressources externes Ressources internes - Environnement local Environnement des affaires - Développement régional}

\begin{abstract}
RÉSUMÉ
Cette étude comparative de l'internationalisation des petites et moyennes entreprises est basée sur une enquête dans quatre régions types de trois pays scandinaves (Finlande, Norvège et Suède), soit 12 régions. L'objectif est de mesurer la signification du milieu local de l'entreprise et l'étendue des relations interfirmes sur le niveau d'internationalisation. Les entreprises sont groupées en trois catégories : exportatrices engagées, exportatrices sporadiques et non
\end{abstract}

\section{LES AUTEURS}

Heikki Eskelinen (économiste) est directeur du Département des sciences sociales de l'Institut Karelien de I'Université de Joensuu, Finlande. Ses principaux domaines de recherche sont la dynamique industrielle, la compétitivité des régions nordiques périphériques et l'impact des interactions transfrontalières.

Eirik Vatne est professeur en géographie économique à l'École d'administration économique et des affaires de Bergen, Norvège. Ses principaux intérêts de recherche sont présentement le développement technologique et les liens entre le global et le local, et les caractéristiques du développement des firmes, leur flexibilité et leur sens de l'innovation. 
exportatrices. En termes de relations verticales et horizontales de coopération, les exportateurs engagés ne se distinguent guère des autres catégories. Les différences entre les régions types et entre les pays sont peu importantes quand il s'agit de l'utilisation des ressources internes dans les entreprises. Les différences dans le recours aux ressources externes sont toutefois plus importantes entre les pays qu'entre les régions. Les meilleurs indicateurs de succès des entreprises comme exportatrices sont le recours aux technologies de production et les types de produits offerts.

\begin{abstract}
The study deals with the internationalization of independant small and mediumsized enterprises located in four different types of local environment in Finland, Norway and Sweden. The focus is on the significance of a local operational environment and interfirm relations in the internationalization process. The firms are divided into committed exporters, experimental exporters and non-exporters. The findings suggest that in terms of horizontal and vertical cooperative relations, the committed exporters are not very dissimilar from the other two groups. Differences by country and by region in internal resources of the firm are also quite insignificant, whereas in the use of external resources there were somewhat more inter-country differences than interregional differences. Production technology and the profile of the products of the firm where the best predictors of success on the export market.
\end{abstract}

\title{
RESUMEN
}

En este estudio se trata la internacionalización de pequeñas y medianas empresas, ubicadas en cuatro tipos de entornos locales en Finlandia, Noruega y Suecia. El trabajo se centra en la importancia del ambiente operacional local $y$ en las vinculaciones entre empresas en el proceso de internacionalización. Las compañías se dividen en exportadores comprometidos, exportadores experimentales y no exportadores. Los resultados del estudio sugieren que en términos de relaciones cooperativas horizontales y verticales, los exportadores comprometidos no se distinguen de los otros dos grupos. Las diferencias entre países o regiones en los recursos internos a la firma son también bastante reducidas, mientras que en el uso de recursos externos habría algo más de diferencia entre países que entre regiones. La tecnología de producción y el perfil de los productos son los mejores indicadores para predecir el éxito como exportadores.

\section{Introduction}

L'effet des échanges internationaux de biens et services sur les différences territoriales et sur la division du travail a longtemps constitué un sujet central de discussion en économie et en géographie économique. En particulier, on s'est arrêté longuement sur l'importance du commerce international pour l'économie de petits pays. Par exemple, les pays nordiques (Danemark, Finlande, Islande, 
Norvège et Suède) sont des petits pays à l'économie beaucoup plus ouverte que les grands pays de l'OCDE.

En accord avec la théorie des avantages comparatifs, l'exportation de ces pays nordiques a été basée avant tout sur l'exploitation des ressources naturelles telles que les minéraux, la forêt, la pêche, le pétrole et l'hydroélectricité. Les avantages compétitifs dynamiques de leurs principales entreprises ont longtemps reposé sur l'évolution de ces industries. Au regard de l'infrastructure industrielle typique de ces pays, l'accent a été mis sur les technologies de procédés, les opérations à large échelle, l'accès aux marchés globaux et les très grandes entreprises (Sölvell, Zander et Porter, 1991).

Toutefois, la croissance rapide du commerce intra-industrie est une caractéristique importante qui a commencé à favoriser la transformation de l'économie. Ce changement est en partie dû à la complexité croissante de l'industrie manufacturière des pays développés, recherchant de plus en plus la «flexibilité » et évoluant vers une "économie réticulaire» dans une période «post-fordiste». Le développement du commerce intra-industrie a permis d'ouvrir des marchés d'exportation pour les petites firmes œuvrant sur des créneaux spécialisés. Toutefois, la plus grande partie de la littérature touchant le rôle des entreprises dans les échanges internationaux continue encore à se limiter aux grandes entreprises et aux multinationales (EMN) (Buckley et Casson, 1985 ou Dunning, 1988).

Cet article porte plutôt sur l'internationalisation des petites et moyennes entreprises finlandaises, suédoises et norvégiennes. Notre analyse essaiera de répondre à la question suivante : pourquoi les PME nordiques sont-elles capables de soutenir la compétition sur des marchés de plus en plus ouverts, notamment à la suite de leur intégration à la Communauté économique européenne et aux multiples développements internationaux ${ }^{1}$ ?

Dans le débat touchant l'avenir des PME des pays nordiques et de toute l'industrie nordique en général, la localisation géographique et les caractéristiques économico-spatiales de ces pays ont reçu beaucoup d'attention. Ces caractéristiques spécifiques sont une faible densité de la population, des infrastructures industrielles particulièrement dispersées et des coûts élevés de transport. Comparées à leurs principaux compétiteurs de l'Europe de l'Ouest, les PME nordiques sont localisées loin du cœur de leur propre marché interne, normalement dans de petites localités.

1. La Finlande et la Suède sont membres de la CEE depuis 1995. La Norvège est liée à ce marché commun par des accords particuliers. 
On peut penser que ces caractéristiques environnementales spécifiques pour leurs opérations ont une importance particulière pour les petites entreprises aux ressources, par définition, limitées. Une PME a peu de possibilités d'influencer son environnement et a besoin de recourir aux ressources externes pour soutenir sa stratégie, par exemple, d'internationalisation. Si, comme on le croit, la qualité, l'accessibilité et le coût des ressources nécessaires sont fonction des particularités structurelles des communautés ou des régions concernées, les PME plus proches des marchés principaux ou des centres plus grands et plus diversifiés devraient accroître leur compétitivité à mesure que leurs activités internationales se développent.

\section{Barrières à l'exportation des PME}

\subsection{Le problème des ressources}

Les recherches antérieures sur l'internationalisation et les comportements à l'exportation ont montré plusieurs différences d'attitudes entre les PME et les grandes entreprises (Cavusgil et Nevin, 1981). Les grandes compagnies mettent en général plus d'énergie sur le marketing et orientent celui-ci de façon plus spécifique vers les besoins des clients internationaux (Sriram et Sapienza, 1991). Parmi les PME, celles dont les ressources sont plus importantes (souvent les moyennes) semblent développer des politiques à l'exportation plus actives par rapport à celles dont les ressources sont plus limitées (Bijmolt et Zwart, 1994). D'un autre côté, beaucoup de petits exportateurs semblent agir de façon réactive dans leurs opérations internationales (Kaynak, Ghauri et Olofsson-Bredenlöw, 1987). Manquant le plus souvent de bonnes connaissances générales sur les marchés étrangers, ils ont des difficultés à juger de leurs capacités compétitives sur ces marchés internationaux et semblent avoir peine à bien orienter leur stratégie d'exportation (Edmunds et Sarkis, 1986 ; Green et Larsen, 1987 ; O'Rourke, 1985). Ainsi, le manque d'information reliée à l'exportation entrave souvent les activités internationales de plusieurs PME (Reid, 1984).

Les PME font aussi face à des défis spéciaux que leur pose une situation financière rendue plus difficile provenant d'équités plus limitées et de profits plus faibles. Elles tendent à avoir plus de problèmes que les grandes entreprises à s'ajuster aux règles étrangères ou internationales et aux standards internationaux, au manque de liaisons d'affaires et de réseaux internationaux, et elles ont des difficultés à organiser la distribution de leurs produits de façon appropriée (Bilkey et Tesar, 1977). Plusieurs de ces problèmes peuvent constituer une «masse critique» pour bloquer les PME (Attiyeh et Wenner, 1979). Le chiffre d'affaires, la valeur ajoutée et les profits per capita sont généralement plus faibles dans les PME (Rothwell et Zegveld, 1982). En outre, les PME 
manquent de spécialistes en marketing pour leurs opérations internationales (Diamantopoulos, 1988).

D'autres recherches récentes ont aussi mis en évidence le fait que les PME ont des problèmes de gestion spécifiques. Le taux d'échec des PME est élevé. Il est vrai que plusieurs petites entreprises préfèrent ne pas grandir (Penrose, 1959); elles ont souvent une stratégie de survie ou un fonctionnement au jour le jour (Boswell, 1973). Plusieurs d'entre elles pensent peu à long terme et, en conséquence, voient mal venir ce qui les menace. Mais même si elles peuvent prévoir les dangers, elles manquent de ressources matérielles et de personnel apte à relever de tels défis efficacement (Hull et Hjern, 1987). Chez beaucoup de petites firmes, l'imitation plus que la planification formelle constitue un comportement habituel.

À la lumière de ces observations, il est donc évident que les PME ne peuvent étendre leurs activités sur le plan international de la même manière que les grandes entreprises. Christensen (1991) a montré qu'il existe deux éléments centraux qui différencient les PME des grandes entreprises à ce propos. Premièrement, l'organisation des PME est moins orientée vers l'exportation ou vers les opérations internationales. On peut faire ce constat par la faible part des ressources internes destinées aux activités d'exportation et par des orientations de la direction et de l'organisation moins tournées vers l'exportation. Deuxièmement, la taille semble affecter la façon dont les firmes recherchent et utilisent l'information sur les marchés internationaux. Les plus grandes entreprises développent plus facilement une stratégie pour obtenir l'information et orienter leur commercialisation, alors que les plus petites firmes semblent plus influencées par les événements et l'expérience.

\subsection{Le management}

Ainsi, la rareté en ressources humaines et matérielles est un important facteur explicatif pour comprendre pourquoi les petites firmes exportent moins que les grandes. Une variable majeure est la qualité du propriétaire-dirigeant. Les petites firmes sont le plus souvent dominées par l'entrepreneur (Imai et Baba, 1991). Souvent perçu comme un avantage, ce facteur se transforme en désavantage par le fait que ce dernier doit partager son temps entre la direction et ses interventions pour contrôler directement la production. Les caractéristiques personnelles du propriétaire-dirigeant, telles que son niveau d'instruction, son expérience de travail, son réseau social et sa personnalité, sont, par conséquent, fortement interreliées avec l'organisation de la firme (Bouwen et Steyaert, 1990).

Bref, les ressources personnelles du dirigeant sont d'une importance cruciale pour les PME. L'habileté de l'entrepreneur à établir et à développer des relations sociales avec d'autres individus dans d'autres firmes devient très 
importante dans le recours à des relations interfirmes. Un bon fonctionnement et la confiance dans les relations sociales peuvent donner accès à toutes sortes de ressources externes, à des informations non disponibles sur le marché et à du savoir-faire spécifique pour améliorer les capacités des PME concernées. D'un autre côté, la position dominante du propriétaire-dirigeant peut aussi être la principale raison du mauvais fonctionnement de la PME (Rainnie, 1989).

\subsection{L'accès aux ressources externes}

En tant qu'organisations économiques, les PME doivent continuellement faire face à la menace de faillite même sur une marché compétitif normal. En général, la survie d'une organisation dépend de sa capacité à acquérir et à maintenir les ressources nécessaires pour répondre au changement dans l'environnement. Cette capacité à diminuer l'incertitude environnementale représente l'efficacité de la firme (Pfeffer et Salancik, 1978).

D'autres aspects de l'interface firme-environnement ont été analysés dans les théories des coûts de transaction, de l'échange social et du management stratégique. Ces recherches traditionnelles ont eu un impact décisif sur diverses applications reliées à l'étude présente (Christensen et al., 1990; Christensen et Lindmark, 1993 ; Vatne, 1995). C'est pourquoi nous ne ferons que résumer ici les principaux points de ces théories.

Les activités et les transactions économiques peuvent être comprises si on les décomposent en séquences de logistique interne, de production et de logistique externe, de commercialisation et autres activités de service, lesquelles forment la chaîne de valeurs. Une firme internalise ses activités aussi longtemps que les coûts pour externaliser ces transactions sont moins importants que ceux qui seraient requis en recourant au marché. En ce sens, internaliser une transaction ou la faire dépendre d'une opération externe dépend de la nature même de la transaction. Lorsqu'une transaction demande des actifs de nature semi-spécifique, la théorie des coûts de transaction prévoit qu'elle relèvera d'accords bilatéraux ou trilatéraux dans des arrangements interorganisationnels qui permettront de sauvegarder les intérêts des parties contractantes (Williamson, 1979; 1985). Ainsi, les économistes néo-institutionnels affirment que la confiance entre les partenaires pourra être institutionnalisée à travers des contrats implicites ou explicites.

La littérature en management stratégique porte aussi un fort intérêt au problème de l'interface organisation-environnement (Porter, 1985). À ce propos, la stratégie centrale d'une firme doit s'appuyer sur ses actifs ayant une spécificité particulière, tels que ses ressources naturelles ou technologiques, ses ressources humaines et son savoir-faire. Toutes ses ressources internes et sa gestion doivent être orientées pour soutenir et développer ces habiletés 
centrales. Pour tirer profit de ces habiletés, la firme doit s'entendre avec ses équipementiers et ses fournisseurs de pièces et de services. Cet apport externe peut être intégré à la firme ou relever de contrats systématiques. Le caractère intangible de ces relations est souvent avancé pour expliquer pourquoi les firmes sont activement engagées dans des relations interorganisationnelles, préférant ainsi des arrangements coopératifs verticaux à l'intégration verticale. En ce sens, la question de la propriété peut être d'une importance secondaire, puisque le contrôle vertical et une plus faible incertitude est ce qui importe d'un point de vue stratégique (Reve, 1990).

Contrairement à Williamson (1985) qui soutenait que le contrat peut être un substitut à la confiance, Granovetter (1985) défend un modèle comportemental et insiste sur le rôle actif des contacts personnels et sur les structures qui permettent de développer des relations interfirmes dynamiques. Un tel modèle est basé sur l'hypothèse qu'un système de valeur particulier est lié à ces relations, l'identification des partenaires étant un facteur critique dans leur formation. Les relations sociales s'établissent d'abord entre des individus et tendent à être durables à long terme. D'un autre côté, les relations des firmes basées sur des contrats relèveront souvent de vues à plus court terme.

De plus, le développement de relations interfirmes repose souvent sur un processus cumulatif d'ajustements, d'investissements et de développements de confiance mutuelle, de liens et de dépendance. Ce processus d'adaptation se développe souvent en apprentissage interactif et permet aux firmes de créer conjointement des nouvelles habiletés croisées intangibles qui sont difficiles à imiter (Johansson et Mattsson, 1987). Selon la théorie des échanges sociaux, les relations sociales évoluent lentement, démarrant à partir de petits faits ou de transactions peu risquées et requérant peu de partage de confiance. Avec le temps, les deux parties développent de la confiance et prennent des engagements plus poussés (Blau, 1968).

Peu d'études sur l'exportation des PME se sont arrêtées à l'impact des stimuli externes pour soutenir l'exportation. Dans la plupart des cas, les influences analysées avaient plutôt un caractère passif. Bon nombre de ces études sont de simples descriptions sur le terrain, relevant de conditions structurelles macroéconomiques sur les marchés nationaux ou étrangers, telles que le niveau de concurrence, la taille minimale et les standards technologiques, les comportements des consommateurs, les règles du commerce, les délais requis de livraison et les risques économiques.

La supposée approche réticulaire en marketing international porte beaucoup plus spécifiquement sur les effets interorganisationnels, mettant l'accent sur le réseautage comme façon d'obtenir une meilleure compréhension du marché, de développer des canaux appropriés de distribution et de créer plus 
systématiquement des produits innovants (Johansson et Mattsson, 1987). Ainsi, ces études ne sont orientées que sur la trajectoire de l'interface firme-marché.

Comme Christensen (1991) le note en se référant à Porter (1985), le processus d'internationalisation d'une firme ne peut être vu qu'à la lumière de la distribution horizontale de la chaîne de valeur, mais il doit aussi être relié aux relations verticales et à l'interface entre le système de support interne et celui de support externe.

\subsection{La proximité sociale et géographique}

En acceptant cette importance des conditions culturelles et sociales dans la création d'interrelations synergiques, la proximité doit être pleinement prise en compte dans le développement de liens interfirmes. Son rôle prend une valeur spéciale dans le cas d'interrelations à base de dialogue extensif ayant pour but de résoudre des problèmes pour finaliser des transactions (Lundvall, 1988). Il est reconnu que les PME sont souvent engagées dans des productions de biens standards répondant à des transactions intra-industries qui nécessitent des communications extensives.

Dans le cas des PME, en particulier, on peut montrer que la proximité non seulement sociale, mais aussi spatiale, devient très importante dans l'offre de savoir-faire, car elle permet aux firmes de participer à des arrangements interfirmes et de développer des relations facilitant des échanges dynamiques et innovants (Harrison, 1992). En ce qui a trait aux plus petites firmes, elles sont impliquées dans des productions locales relevant de facteurs liés aux spécialités disponibles et au niveau d'éducation de la main-d'œuvre, aux institutions de $\mathrm{R}-\mathrm{D}$, aux moyens de communication, aux infrastructures publiques et à la texture de l'environnement industriel.

Les théoriciens des « districts industriels », de Marshall (1896) à Garofoli (1991), expliquent le dynamisme des PME concentrées spatialement et la croissance d'une industrie par la proximité sociale et géographique résultant de la division extensive du travail. La culture contingente entrepreneuriale qui s'est développée historiquement, renforcée par la disponibilité des économies externes, semble donc une caractéristique structurelle distinctive de ces districts industriels.

Il y a toutefois beaucoup de transactions qui ne requièrent pas la proximité. Ainsi, les opérations standardisées qui ont moins besoin de dialogues et d'échanges d'information peuvent très bien relever de relations de routine ou d'accords contractuels et peuvent donc être facilement conclues à distance (Scott, 1988). Pour les PME, compte tenu de leurs faibles ressources internes et de leur implantation dans leur milieu d'affaire local, le rôle des relations sociales devient plus important que les accords contractuels. En considérant 
l'importance particulière que revêt la confiance chez les PME, la proximité avec des partenaires d'affaires devient cruciale pour les relations tant horizontales que verticales.

\subsection{Un modèle pour une étude empirique}

À partir de ce que nous venons de discuter, on peut conclure que les PME aux ressources limitées sont fortement dépendantes de leur environnement et de l'accès aux ressources externes pour compenser le manque de ressources de base. Le développement réussi des PME repose présumément sur une spécialisation et une concentration autour du métier central, ainsi que sur le recours aux ressources externes lorsqu'on doit réaliser des travaux spéciaux de production, de services ou de distribution et de commercialisation. Le propriétaire-dirigeant joue un rôle crucial dans l'établissement et le développement de contacts avec les partenaires externes, spécialement dans des relations dynamiques interfirmes basées sur des échanges en toute confiance d'informations sensibles et d'engagements d'actifs spécifiques à investir.

La dépendance à des relations dynamiques pour faciliter les échanges sociaux et le besoin de confiance rehaussent l'importance de la proximité et de la localisation. Par exemple, un environnement d'affaires en pleine expansion peut donner un meilleur accès à de l'information clé sur la technologie et sur les changements dans le marché, ainsi qu'à des consultants, sous-traitants et distributeurs plus compétents, et ce, à un coût moindre.

Selon la théorie d'agglomération, on peut alors avancer l'idée que les PME profitant d'un environnement d'affaires plus large et plus diversifié ont plus de chances d'obtenir des avantages compétitifs sur les PME établies dans des milieux ruraux. Les conditions de localisation influencent aussi l'offre des facteurs de production et ainsi les ressources internes des PME, comme on l'a mentionné à propos de la main-d'œuvre et de sa spécialisation. De même, on peut argumenter que la localisation a aussi un effet sur les qualifications de l'entrepreneur ou du dirigeant, son expérience et son réseau social. En particulier, l'habileté de l'entrepreneur à contrôler l'interface organisationenvironnement aussi bien que les relations sociales dans un contexte international est, en effet, un aspect crucial du succès de l'internationalisation.

Même en délaissant les arguments mettant l'accent sur le rôle des ressources externes et de celles du milieu local dans l'internationalisation des PME, il est évident que ces firmes sont avant tout, et surtout, dépendantes de leurs propres ressources internes et de leurs produits pour gagner des parts sur les marchés étrangers. La figure 1 résume les divers éléments du modèle développé par Vatne (1995). 
FIGURE 1

Un modèle conceptuel expliquant l'internationalisation des PME

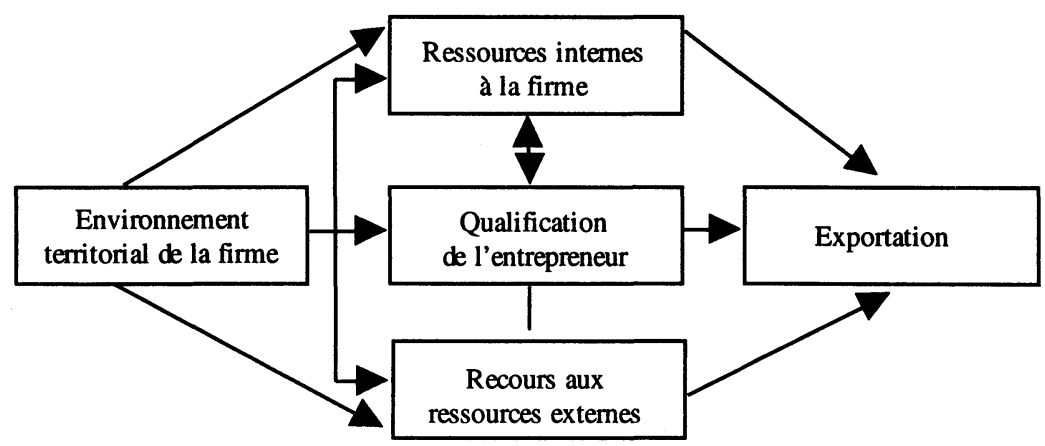

Dans les sections suivantes, l'analyse empirique dérivée de ce modèle s'arrêtera particulièrement sur les ressources des PME selon leurs différents niveaux d'internationalisation et leur dépendance à l'égard de leur environnement économique. Mais comme le niveau d'internationalisation s'explique aussi par l'industrie à laquelle les PME appartiennent, nous nous sommes limités, dans cette étude, aux seules entreprises de l'industrie de la transformation du bois et du métal. Ce sont des industries qui exportent une bonne part de leur production et ce, dans beaucoup de pays.

Dans cet article, notre analyse se résumera à des statistiques descriptives bivariées et à un test de signification portant sur les différences entre trois groupes de firmes : celles qui sont fortement engagées vers l'exportation, celles qui exportent de façon sporadique et celles qui n'exportent pas du tout. Bien que la généralisation de telles données soit limitée, ces dernières peuvent fournir diverses indications sur les processus pouvant expliquer les comportements des firmes exportatrices.

\section{Firmes internationales et firmes limitées au marché national}

Les données ont été recueillies à partir d'une enquête effectuée auprès de 274 entreprises indépendantes, ayant entre 10 et 199 employés, de l'industrie de la transformation du bois et de celle de la transformation du métal ${ }^{2}$. Le tableau 1 donne quelques caractéristiques de cet échantillon.

2. Selon la nomenclature du NACE, ce sont les industries $n^{\text {os }} 20,22,24$ et 27 à 36 . Suivant la nomenclature ISIC, il s'agit des $n^{\text {os }} 331,332$ et 381 à 385 . 
TABleau 1

Quelques données sur l'échantillon

\begin{tabular}{lcccc}
\hline & Norvège & Finlande & Suède & Total \\
\hline Nombre de firmes & 93 & 80 & 101 & 274 \\
-industrie du bois (\%) & $42 \%$ & $29 \%$ & $35 \%$ & $35 \%$ \\
-industrie métallique (\%) & $58 \%$ & $71 \%$ & $65 \%$ & $65 \%$ \\
Taille: & & & & \\
- employés : nombre moyen & 24 & 19 & 21 & 21 \\
Part d'exportateurs (\%) & $58 \%$ & $45 \%$ & $60 \%$ & $55 \%$ \\
\hline
\end{tabular}

Trente-cinq pour cent de ces PME œuvrent dans l'industrie du bois. Le nombre moyen d'employés était de 21 en 1991. Plus de la moitié de ces firmes exportaient cette année-là, dont seulement quelques-unes avaient développé leur exportation. Pour cette raison, on doit bien voir la portée limitée de l'idée d'internationalisation dans cet échantillon.

Les PME étudiées ici étaient localisées dans quatre types de régions de la Finlande, de la Norvège et de la Suède. Ces régions diffèrent beaucoup au regard de la taille, de la localisation et des structures (grandes villes, villes industrielles, petites villes et zones rurales périphériques). Par exemple, les grandes villes ont entre 200000 et 500000 habitants, alors que les villes industrielles en ont environ 100000 . Pour leur part, les petites villes se limitent à environ 20000 habitants et les zones rurales périphériques sont composées de quelques villages. Au total, ces 12 régions (4 par pays) forment un environnement suffisamment varié pour analyser comment l'environnement opérationnel local a un effet sur l'internationalisation.

On pourrait ainsi diviser grosso modo les PME en deux groupes : celles qui exportent et celles qui se limitent au marché national. En pratique, le niveau d'internationalisation est beaucoup plus varié que cela. Certaines firmes exportent toute leur production alors que d'autres n'en exportent qu'une petite partie. Parmi ces dernières, on en trouve aussi qui ne sont pas intéressées à augmenter leurs exportations ou à développer de nouvelles opérations internationales.

Nous avons donc divisé les PME exportatrices entre celles qui sont fortement engagées vers l'exportation et celles qui le sont de manière expérimentale ou sporadique. Ces deux groupes constituent une version simplifiée de la typologie développée par Cavusgil (1984). Les PME qui sont «engagées » dans l'exportation exportent au moins $20 \%$ de leur production et exportent vers au moins trois pays. Celles qui exportent moins de $20 \%$ de leur production et dans moins de deux pays sont considérées comme des exportatrices «sporadiques ». Le tableau 2 donne quelques chiffres sur ces deux groupes. 
TABLEAU 2

Répartition des PME nordiques $(\mathrm{N}=\mathbf{2 7 4})$

entre les exportatrices «engagées», les «sporadiques» et celles qui n'exportent pas, selon les régions et les pays, en pourcentage

\begin{tabular}{lccc}
\hline & $\begin{array}{c}\text { Exportatrices } \\
\text { engagées } \\
(\boldsymbol{\%})\end{array}$ & $\begin{array}{c}\text { Exportatrices } \\
\text { sporadiques } \\
(\boldsymbol{\%})\end{array}$ & $\begin{array}{c}\text { Non- } \\
\text { exportatrices } \\
(\boldsymbol{\%})\end{array}$ \\
\hline Norvège $(\mathrm{N}=93)$ & 37 & 26 & 38 \\
Finlande $(\mathrm{N}=80)$ & 31 & 14 & 55 \\
Suède $(\mathrm{N}=101)$ & 50 & 11 & 40 \\
Industrie du bois $(\mathrm{N}=97)$ & 34 & 20 & 46 \\
Industrie du métal $(\mathrm{N}=177)$ & 43 & 15 & 42 \\
Exportatrices $(\mathrm{N}=155)$ & 70 & 30 & 43 \\
Total $(\mathrm{N}=274)$ & 40 & 17 & \\
\hline
\end{tabular}

Environ deux cinquièmes des PME peuvent être considérées comme des exportatrices engagées et un peu moins d'un cinquième comme sporadiques. Les non-exportatrices, soit 117 entreprises, sont toutefois les plus nombreuses. On trouve une différence marquée dans la répartition de ces trois groupes selon les pays. Ainsi, la part des PME exportatrices engagées est la plus élevée en Suède, soit là où l'industrie manufacturière a une longue tradition dans les exportations. Compte tenu de leur proximité géographique et culturelle avec la Suède et les faibles barrières douanières, les PME norvégiennes, pour leur part, sont plutôt orientées vers le marché suédois et on retrouve chez celles-ci une assez grande proportion de firmes exportatrices expérimentales. Enfin, en Finlande, la distance linguistique et géographique entre les autres pays nordiques et les pays de l'Europe occidentale constitue une barrière d'échange effective. Ainsi, plus de la moitié des PME présentes dans l'échantillon sont des non-exportatrices. Par contre, les différences par secteurs entre les trois groupes de PME ne sont pas aussi grandes que les différences par pays.

Environ $43 \%$ du chiffre d'affaires total des firmes exportatrices engagées provenait des exportations en 1991 alors que ce pourcentage n'était que d'environ $7 \%$ pour les exportatrices sporadiques. Pour diverses raisons, la part des ventes locales (dans les districts de marché de travail) était plus faible pour les firmes exportatrices engagées (moins de $10 \%$ ) que pour le deuxième groupe (environ $20 \%$ ). Les ventes des PME non exportatrices étaient distribuées à peu près également entre le marché local et le reste du pays. De même, les différences sur le plan de la profitabilité entre les trois groupes étaient faibles.

En suivant l'argumentation développée à la section 2, l'internationalisation peut être vue comme demandant des ressources importantes à l'interne 
ou une recherche active de ressources externes. Nous nous arrêterons d'abord sur une comparaison entre les trois pays nordiques de la mobilisation par les PME des ressources internes. Nous utiliserons pour cela une simple analyse de variance, avec éventuellement un test de chi-carré pour les données nominales. Par la suite, nous étudierons les différentes façons utilisées par les PME pour obtenir les ressources externes.

\subsection{L'utilisation des ressources internes}

Comme on le sait, les ressources humaines et monétaires de chaque firme dépendent considérablement de sa taille. De même, la qualité de ces ressources (par exemple, la compétence des employés) et leur compatibilité avec le métier de la firme sont aussi cruciales. En outre, des recherches antérieures ont montré qu'une des ressources clés réside en la personne du propriétaire-dirigeant ; la continuité et le développement des activités de chaque firme dépend en bonne partie de lui (voir la section 2). Enfin, la technologie et le type d'organisation peuvent être considérés comme importants puisqu'ils influent sur l'efficacité des ressources humaines et monétaires.

Le tableau 3 résume les différences des ressources internes entre les trois groupes étudiés. Ce tableau montre la valeur moyenne des différentes variables mesurant les ressources internes dans les firmes pour chacun des groupes de PME exportatrices ou non, et indique si ces différences sont significatives statistiquement.

Les firmes considérées comme des exportatrices engagées sont généralement plus grandes que les sporadiques et les non-exportatrices tant au regard du chiffre d'affaires que du nombre d'employés. Selon les entrepreneurs, leurs employés sont plus spécialisés et leur niveau technologique ou d'automatisation est plus élevé. De plus, elles ont un pourcentage plus élevé de cols blancs, le niveau de scolarité de leurs employés a augmenté dans les années récentes et les propriétaires-dirigeants sont plus compétents en ce qui concerne la formation, les langues connues et l'expérience avec des firmes étrangères et autres secteurs comparés à leurs confrères des autres groupes. Par contre, les différences ne sont pas significatives entre les trois groupes de PME quant à la formation, à l'expérience de travail et à la maîtrise des langues étrangères des cadres. En fait, les PME non exportatrices sont même plus fortes de ce côté que les exportatrices sporadiques.

La plupart du temps, ce sont ces PME non exportatrices qui sont significativement différentes des deux autres groupes. Les exportatrices sporadiques semblent se situer entre les deux autres groupes quant à l'accès aux ressources internes. 
Tableau 3

Les différences de ressources internes

selon que les PME sont exportatrices engagées, exportatrices sporadiques ou non exportatrices, en 1991

\begin{tabular}{|c|c|c|c|c|}
\hline & $\begin{array}{l}\text { Xxportatrices } \\
\text { engagées }\end{array}$ & $\begin{array}{l}\text { Exportatrices } \\
\text { sporadiques }\end{array}$ & $\begin{array}{c}\text { Non- } \\
\text { exportatrices }\end{array}$ & $\begin{array}{l}\text { Niveau de } \\
\text { signification }\end{array}$ \\
\hline \multicolumn{5}{|l|}{$\begin{array}{l}\text { Taille } \\
\text { - nombre d'emplovés }\end{array}$} \\
\hline - médiane & 30 & 29 & 16 & ** \\
\hline - moyenne & 45 & 39 & 24 & ** \\
\hline \multicolumn{5}{|l|}{$\begin{array}{l}\text { - chiffre d'affaires } \\
\text { (millions de MF) }\end{array}$} \\
\hline - médiane & 17 & 10 & 6 & ** \\
\hline - moyenne & 24 & 16 & 11 & ** \\
\hline \multicolumn{5}{|l|}{ Organisation du travail } \\
\hline \multicolumn{5}{|l|}{ - processus de production selon } \\
\hline \multicolumn{5}{|l|}{ Moyenne (échelle de 1 à 5 ) } \\
\hline - niveau technologique & 2,9 & 2,7 & 2,3 & ** \\
\hline - niveau d'automatisation & 2,6 & 2,2 & 1,7 & ** \\
\hline \multicolumn{5}{|l|}{$\begin{array}{l}\text { Qualité des entrepreneurs } \\
\text { Expérience (en \%) } \\
\text { des entrepreneurs }\end{array}$} \\
\hline - grandes firmes & 49 & 47 & & \\
\hline - firmes conseils & 19 & 20 & 13 & \\
\hline - firmes étrangères & 32 & 29 & 16 & $*$ \\
\hline - autres secteurs & 66 & 47 & 47 & ** \\
\hline \multicolumn{5}{|l|}{$\begin{array}{l}\text { - niveau de scolarité supérieur } \\
\text { du personnel technique } \\
\text { et commercial } \\
\text { (en \%) des firmes }\end{array}$} \\
\hline - au moins 4 années & 23 & 21 & 9 & \\
\hline $\begin{array}{l}-2 \text { à } 3 \text { années } \\
- \text { moins de } 2 \text { ans }\end{array}$ & $\begin{array}{l}36 \\
39\end{array}$ & $\begin{array}{l}41 \\
56\end{array}$ & & \\
\hline \multicolumn{5}{|l|}{$\begin{array}{l}\text { - langues maîtrisées } \\
\text { (en \%) des entrepreneurs }\end{array}$} \\
\hline - anglais & 87 & 84 & 66 & ** \\
\hline - allemand & 60 & 44 & 29 & ** \\
\hline \multicolumn{5}{|l|}{ Type d'employés } \\
\hline - part des cols blancs (en \%) & 29 & 22 & 20 & $* *$ \\
\hline - changements dans le niveau & & & & \\
\hline \multicolumn{5}{|l|}{$\begin{array}{l}\text { - niveau de scolarité (en \%) } \\
\text { des cadres }\end{array}$} \\
\hline - université ou équivalent & 14 & 12 & 13 & \\
\hline - collégial & 37 & 24 & 30 & \\
\hline \multicolumn{5}{|l|}{$\begin{array}{l}\text { - expérience de travail (en \%) } \\
\text { des cadres }\end{array}$} \\
\hline - grandes firmes & 20 & 21 & & \\
\hline - firmes conseils & 6 & 3 & 4 & \\
\hline - firmes étrangères & 10 & 6 & 9 & \\
\hline \multicolumn{5}{|l|}{ - langues maîtrisées (en \%) } \\
\hline - anglais & 58 & 46 & 49 & \\
\hline - allemand & 22 & 12 & 16 & * \\
\hline
\end{tabular}

$* 0,05 ; * * 0,01$. Niveau de signification (corrélation croisée, $c^{2}$, analyse de variance à un seul tour, test t). 
Bref, ces premiers résultats appuient l'hypothèse selon laquelle la qualité des ressources internes des firmes et de leur dirigeant a un impact majeur dans le processus d'internationalisation des PME. Mais il faut être prudent avec ces données, puisqu'elles relèvent d'une simple analyse statistique bivariée ${ }^{3}$. En particulier, on doit noter que les chiffres que l'on vient de présenter sont basés sur de simples données croisées et, par conséquent, il est difficile d'en tirer des généralisations permettant d'affirmer que la présence de fortes ressources internes chez les PME exportatrices engagées est la cause plutôt que le résultat de leur internationalisation.

\subsection{Les ressources externes}

Comme nous l'avons vu, il existe plusieurs possibilités pour mobiliser des ressources externes capables de soutenir les stratégies des PME exportatrices. Par exemple, elles peuvent conclure des transactions traditionnelles sur le marché pour obtenir divers services. En plus de ces achats, les PME peuvent entre autres choses développer des relations de coopération de long terme ou entrer dans des réseaux pour développer des produits, commercialiser ou sous-traiter. Il est aussi possible que leurs liens d'affaires et leurs relations d'affaires complètent ces réseaux, puisque l'on sait que les contacts personnels jouent un rôle prépondérant dans la recherche d'information et le maintien ou l'amélioration de la compétitivité. En général, l'accès croissant à des ressources externes est un processus dynamique dans lequel les ressources internes des firmes, leur environnement d'affaires, leurs réseaux, la position sociale de l'entrepreneur et sa capacité à résoudre les problèmes sont intimement liés. Le tableau 4 présente divers résultats touchant les moyennes en ce qui concerne l'accès à ces ressources externes.

Dans l'ensemble, ces données ne permettent pas de conclure que les PME exportatrices sont plus actives que les non-exportatrices dans l'acquisition de ressources externes. Les différences entre les trois groupes de PME dans cette recherche de ressources externes ne sont pas aussi importantes et systématiques que dans le cas des ressources internes.

Par ailleurs, une attention spéciale doit être portée aux contacts des entrepreneurs et à leurs efforts pour développer diverses coopérations interfirmes. On a demandé aux entrepreneurs de décrire leurs liens professionnels et sociaux et leurs accords de coopération. En entrevue, on leur a demandé d'évaluer la qualité de leurs relations de coopération (par exemple, l'importance de leurs recours au marché par rapport à leurs transactions dans le cadre de diverses coopérations).

3. Notons, cependant, que les conclusions demeurent à peu près les mêmes avec une analyse multivariée. 
TABleau 4

Le recours aux ressources externes. PME exportatrices engagées, exportatrices sporadiques et non exportatrices en 1991

\begin{tabular}{|c|c|c|c|c|}
\hline & $\begin{array}{l}\text { Exportatrices } \\
\text { engagées }\end{array}$ & $\begin{array}{l}\text { Exportatrices } \\
\text { sporadiques }\end{array}$ & $\begin{array}{c}\text { Non- } \\
\text { exportatrices }\end{array}$ & $\begin{array}{l}\text { Niveau de } \\
\text { signification }\end{array}$ \\
\hline \multicolumn{5}{|l|}{$\begin{array}{l}\text { Contacts au moins } \\
\text { un fois par semaine } \\
\text { (en \% des entrepreneurs) }\end{array}$} \\
\hline - contacts professionnels locaux & 55 & 60 & 55 & \\
\hline - contacts sociaux locaux & 46 & 39 & 41 & \\
\hline $\begin{array}{l}\text { Contact au moins } \\
\text { une fois par mois } \\
\text { (en \% des entrepreneurs) }\end{array}$ & & & & \\
\hline - de sous-traitants & 57 & 78 & 57 & * \\
\hline - avec des compétiteurs & 32 & 38 & 31 & \\
\hline - avec des clients & 80 & 82 & 70 & \\
\hline - avec d'autres organisations & 41 & 35 & 29 & \\
\hline \multicolumn{5}{|l|}{ Relations de coopération } \\
\hline $\begin{array}{l}\text { - nombre en moyenne } \\
\text { - localisation des plus } \\
\text { importants partenaires }\end{array}$ & 2,2 & 2,3 & 2,0 & \\
\hline Aires locales (en \% des PME) & 30 & 47 & 54 & ** \\
\hline Aires plus larges (en \% des PME) & 24 & 3 & 2 & \\
\hline \multicolumn{5}{|l|}{$\begin{array}{l}\text { Formes de coopération } \\
\text { (en \% des PME) }\end{array}$} \\
\hline - coentreprises & 31 & 30 & 25 & \\
\hline $\begin{array}{l}\text { - contrats de coopération } \\
\text { de long terme }\end{array}$ & 55 & 52 & 47 & \\
\hline - coopération informelle & 58 & 61 & 65 & \\
\hline $\begin{array}{l}\text { - coopération informelle } \\
\text { occasionnelle }\end{array}$ & 75 & 80 & 76 & \\
\hline \multicolumn{5}{|l|}{$\begin{array}{l}\text { Sous-traitance (par rapport } \\
\text { au chiffre d'affaires, } \\
\text { en moyenne, en pourcentage) }\end{array}$} \\
\hline $\begin{array}{l}\text { - sous-traitance venant du local } \\
\text { - sous-traitance venant }\end{array}$ & 9 & 13 & 8 & \\
\hline hors du local & 13 & 6 & 8 & * \\
\hline - sous-traitance livrée au local & 7 & 12 & 36 & $* *$ \\
\hline $\begin{array}{l}\text { - soust-trantance } \\
\text { livrée hors du local }\end{array}$ & 39 & 33 & 29 & \\
\hline \multicolumn{5}{|l|}{$\begin{array}{l}\text { Services d'affaires } \\
\text { (part des achats par les PME } \\
\text { localement, en pourcentage) }\end{array}$} \\
\hline - comptabilité et tenue de livre & 84 & 85 & 90 & \\
\hline - informatique & 75 & 80 & 76 & \\
\hline - publicité et commercialisation & 65 & 70 & 70 & \\
\hline - transport & 76 & 76 & 79 & \\
\hline - conseil en gestion & 49 & 45 & 44 & \\
\hline - conseil en technologie & 55 & 70 & 61 & \\
\hline - services légaux & 72 & 76 & 76 & \\
\hline - services de formation & 53 & 68 & 68 & \\
\hline - services financiers & 84 & 89 & 94 & \\
\hline - services publics aux firmes & 57 & 64 & 42 & \\
\hline
\end{tabular}

$* 0,05 ; * * 0,01$.

Différence significative statistique (tabulation croisée, $c^{2}$, ou analyse de variance à un tour, test $t$ ). 
On constate que les différences entre les trois groupes quant à la participation à des réseaux sociaux d'entrepreneurs sont légères. Un peu plus de la moitié des entrepreneurs ont des rencontres hebdomadaires avec leurs collègues locaux afin de discuter de problèmes professionnels, et moins de $50 \%$, pour des problèmes sociaux. Quant aux recours par les entrepreneurs à leurs soustraitants, à leurs compétiteurs, à leurs clients ou à d'autres acteurs comme source informationnelle, la seule différence statistiquement significative est que les exportateurs sporadiques sont plus souvent en contact avec leurs soustraitants que les exportateurs engagés et les non-exportateurs. De même, une plus grande proportion d'exportateurs que de non-exportateurs estiment avoir des contacts au moins mensuels avec des concurrents, des clients et autres acteurs économiques (tels que les gouvernements); mais la différence n'est pas statistiquement significative. Enfin, les données semblent indiquer que les exportateurs expérimentaux sont le groupe le plus engagé dans des relations sociales externes dans le but de trouver des informations importantes pour l'entreprise.

Les firmes ont déclaré qu'elles participaient en moyenne à un peu plus de deux types de relations réticulaires interfirmes relativement permanentes. Les seules différences statistiquement significatives à ce propos entre les trois groupes touchaient la localisation des plus importants partenaires. Au moins un quart des exportateurs engagés avaient un partenaire étranger alors que c'était très peu le cas pour les autres PME. Les données révèlent en outre que les PME non exportatrices tendent à être beaucoup plus engagées dans des relations interfirmes locales. Cette différenciation s'applique aussi quant au deuxième et au troisième partenaire plus important.

Dans le cas de la sous-traitance comme forme de coopération, les nonexportateurs livrent plus à d'autres firmes locales, alors que les exportateurs engagés recourent plus souvent à des sous-traitants non locaux. Cette différence provient de la place des entreprises dans la chaîne de valeur. Chez les exportateurs engagés, $79 \%$ ont d'abord leurs propres produits ${ }^{4}$, alors que cette part est de $73 \%$ dans le cas des exportateurs sporadiques et de $51 \%$ dans celui des non-exportateurs.

Nous pouvons en outre voir au tableau 4 que plus une firme exporte, plus ses accords de coopération sont formalisés. En d'autres mots, les PME avec peu de relations étrangères, et parfois aucune, ont des relations interfirmes plutôt informelles et souvent occasionnelles. Mais encore ici, les différences entre les trois groupes sont légères et ne sont pas statistiquement significatives.

4. La part de leur production propre sur le chiffre d'affaires dépasse les $50 \%$. 
En moyenne, les PME exportatrices achètent un peu plus de services d'affaires, mais la différence avec les non-exportatrices n'est pas significative. Comme on peut le voir au tableau 4, il n'y a pas de différences systématiques entre les exportateurs engagés, les sporadiques et les non-exporateurs dans le sens qu'aucun de ces groupes n'utilise plus les services d'affaires locaux que les autres. Par contre, les exportateurs engagés semblent moins dépendants des services externes que ceux des autres groupes.

Même si nous ne trouvons pas de différences significatives entre les trois groupes, les données indiquent que les exportateurs engagés recourent plus à leurs ressources internes pour leurs services d'affaires, sont plus activement impliqués dans des relations interfirmes ou de la sous-traitance non locales et tendent à formaliser ces relations. Cela peut s'expliquer partiellement par des ressources internes fort différentes selon les trois groupes. Les PME exportatrices engagées sont plus grandes, ont une production à économie d'échelle plus routinière, ont une capacité interne particulière à planifier et à gérer leurs relations externes et peuvent aisément faire face au problème d'augmentation du risque et des coûts de transaction de nouveaux marchés. Les firmes exportatrices sporadiques, pour leur part, ne semblent pas riches de la même qualité de ressources internes et sont généralement plus impliquées dans des situations imprévues où un risque élevé et un fonctionnement par essais et erreurs caractérisent leurs activités. Si c'est le cas, il semble normal que ces firmes recherchent plus d'informations dans l'environnement, soient plus impliquées dans des coopérations externes et recourent à des services externes de façon plus intensive que leurs concurrents mieux établis.

On a demandé aux entrepreneurs de décrire les motifs et la forme de leurs relations de coopération les plus importantes. Leurs réponses ont été résumées au tableau 5 .

Les motifs pour la coopération diffèrent de façon significative de deux manières. La promotion des exportations comme motif important pour les firmes internationalisées peut être regardée comme évidente. En plus, les deux groupes de firmes exportatrices insistent davantage sur le développement de procédés comme motif pour engager des coopérations. Quant aux formes de coopération, on relève plus de différences entre les trois groupes de PME. Celles touchant l'amélioration du processus de production, le développement de produits, la sous-traitance, la diffusion de l'information technologique et les délais et conditions de livraison sont significativement différentes. En général, les deux groupes de firmes exportatrices accordent plus d'importance à toutes les formes de coopération comparés au groupe de firmes non exportatrices. Il est aussi intéressant de voir que les exportateurs sporadiques, de plusieurs manières, semblent donner plus d'importance aux accords de coopération que les exportateurs engagés. 


\section{TABLEAU 5}

Qualités des relations de coopération les plus importantes (échelle moyenne de 1 à 5 )

\begin{tabular}{|c|c|c|c|c|}
\hline & $\begin{array}{l}\text { portatrices } \\
\text { ngagées }\end{array}$ & $\begin{array}{l}\text { Exportatrices } \\
\text { sporadiques }\end{array}$ & $\begin{array}{c}\text { Non- } \\
\text { exportatrices }\end{array}$ & $\begin{array}{c}\text { Niveau de } \\
\text { signification }\end{array}$ \\
\hline \multicolumn{5}{|l|}{ Motifs } \\
\hline \multicolumn{5}{|l|}{ Capacité à résoudre } \\
\hline les problèmes & 2,67 & 2,59 & 2,70 & \\
\hline Économies d'échelle & 2,87 & 2,63 & 2,45 & \\
\hline Développement de produit & 2,86 & 3,00 & 2,67 & \\
\hline Développement de procédés & 2,78 & 2,73 & 2,24 & * \\
\hline Capacité de livraison & 3,13 & 2,95 & 2,89 & \\
\hline \multirow{2}{*}{\multicolumn{5}{|c|}{ Importance de créneau }} \\
\hline & & & & \\
\hline sur son marché & 2,32 & 2,53 & 2.17 & \\
\hline Promotion à l'exportation & 3,02 & 3,07 & 2,07 & ** \\
\hline \multicolumn{5}{|l|}{ Formes } \\
\hline Visites des partenaires & 3,48 & 3,78 & 3,32 & \\
\hline \multicolumn{5}{|l|}{ Suivi des performances } \\
\hline des partenaires & 2,94 & 3,03 & 2,61 & \\
\hline \multicolumn{5}{|l|}{ Amélioration des procédés } \\
\hline de production & 3,19 & 2,50 & $* *$ & \\
\hline Développement de produits & 3,55 & 3,45 & 3,02 & * \\
\hline Sous-traitance & 2,46 & 3,29 & 2,94 & * \\
\hline \multicolumn{5}{|l|}{ Concordance entre les } \\
\hline Diffusion de l'information & 3,11 & 3,06 & 2,61 & * \\
\hline \multicolumn{2}{|l|}{ Diffusion de l'information } & 3,45 & 3,16 & \\
\hline \multicolumn{5}{|l|}{ Délais et conditions } \\
\hline de livraison & 3,00 & 2,94 & 2,11 & ** \\
\hline Mise en marché conjointe & 2,82 & 3,19 & 2,70 & \\
\hline
\end{tabular}

$* 0,05 ; * * 0,01$

(analyse de variance à deux tours, test t)

\section{Les exportateurs engagés par pays et par région}

Nous venons de montrer les plus importances différences en ressources internes et en ressources potentielles provenant de l'extérieur des PME nordiques selon les divers niveaux d'internationalisation. Dans ce qui suit, la comparaison sera limitée aux ressources des PME les plus internationalisées, soit celles appelées exportatrices engagées. Tant l'environnement opérationnel national que local semble marquer les caractéristiques de ces firmes. En se limitant aux marchés de l'Europe de l'Ouest, ces deux milieux peuvent être vus comme des niveaux élargis conditionnant les activités internationales des PME nordiques.

En ce qui concerne les différences de chaque pays, divers facteurs potentiellement importants présentent des différences dans leurs structures 
industrielles et leur performance économique. Il est vrai que les trois pays nordiques ont certains points en commun. Ils ont tous des économies très ouvertes depuis longtemps et leurs structures de production se sont ajustées à l'évolution de l'économie des pays d'Europe de l'Ouest et ainsi aux conditions des échanges internationaux. Mais parmi ces trois pays, la Norvège s'est spécialisée dans l'exportation de ses ressources naturelles, alors que la Suède a misé davantage sur le commerce interindustries que ses autres partenaires nordiques (voir, par exemple, Lundberg, 1992). Ces différences structurelles s'expliquent par la dotation différente en ressources naturelles et par une industrialisation particulière. De plus, ces pays ont créé des pratiques et ont mis sur pied des institutions spécifiques pour soutenir les échanges internationaux qui se sont reflétées par la suite sur les PME. Quant à la performance industrielle et macro-économique récente des trois pays, elle n'est pas très intéressante. La récession du début de 1990 a été particulièrement dure en Finlande, ce qui se reflète d'ailleurs dans notre analyse.

Du côté des différences régionales, en distinguant les grandes villes, les villes industrielles, les zones de petites villes industrielles et la périphérie rurale, ces différences se perpétuent dans chaque pays nordique. Même si vues sous certains angles, plusieurs régions se ressemblent par leurs caractéristiques structurelles et par leurs rôles dans la division régionale du travail, finalement elles diffèrent toutes. Ces quatre milieux locaux, considérés dans notre analyse comme des marchés de travail spécifiques, peuvent être vus comme offrant aux PME, et spécialement aux PME internationales, différentes conditions et ressources. Ces différences, parmi d'autres, touchent la distance et ainsi les coûts de transport, la densité de population, la taille du marché local et de l'offre de travail, les services d'affaires et de sous-traitance.

La proportion d'exportateurs engagés (de même que celle des autres exportateurs) est plus faible en Finlande qu'en Norvège et en Suède, comme on l'a vu au tableau 2 ; mais les PME finlandaises exportent pour une part plus élevée de leur chiffre d'affaires. En 1991, en Finlande, les exportateurs engagés ont vendu pour environ $53 \%$ de leur chiffre d'affaires total à l'étranger, alors que ce pourcentage n'était que de $42 \%$ en Suède et $39 \%$ en Norvège. Par contre, les différences interrégionales, dans ce cas, étaient plus faibles. Ainsi, la part la plus faible était de $39 \%$ dans les zones de petites villes industrielles et la plus élevée, $50 \%$, dans les grandes villes. Dans les villes industrielles et les zones rurales, les pourcentages étaient respectivement de $40 \%$ et de $44 \%$. Il ne faut pas se surprendre de voir que ces différences sont reliées au rôle des firmes dans la chaîne de valeur : la part des PME offrant leurs propres produits est plus faible dans les petites villes.

On trouve peu de différences entre les pays et les régions dans le niveau de ressources internes des exportateurs engagés. Les entrepreneurs suédois 
évaluent le niveau technologique de leur firme et leur formation comme plus faible, mais considèrent, à l'instar des entrepreneurs finlandais, qu'un plus fort pourcentage de leurs cadres ont une formation universitaire, comparé à la Norvège. La seule différence interrégionale significative quant aux caractéristiques des employés concerne les changements dans le niveau de scolarité. Ce dernier a crû de $91 \%$ chez les exportateurs engagés dans les petites villes industrielles, mais seulement de $39 \%$ dans les grandes villes. On pourrait peutêtre expliquer cela par le fait que les petites villes se sont fortement restructurées récemment, de façon à améliorer la disponibilité d'une main-d'œuvre qualifiée. En général, les différences entre ces pays relativement petits et leurs régions quant aux ressources internes peut provenir d'une homogénéisation croissante due aux pressions de la concurrence chez les exportateurs engagés. On trouve un effet de rétroaction important en cours ici : l'amélioration des ressources nécessaires pour soutenir l'exportation permet d'augmenter les opérations sur un marché européen de plus en plus compétitif.

Le tableau 6 résume ces différents aspects du profil des ressources selon les différents exportateurs engagés.

On relève ainsi plus de différences nationales ou régionales dans les caractéristiques touchant les ressources externes que dans celles portant sur les ressources internes. Toutefois, même si l'offre de ressources pour les opérations internationales des PME peut être considérée comme variant au moins en fonction du type de région, les différences, comme on peut le voir au tableau 6 , ne semblent pas liées de manière linéaire à aucun schéma hiérarchique. En effet, les facteurs appartenant à chaque pays semblent jouer un rôle plus important que ceux appartenant aux régions. En moyenne, les exportateurs engagés de Norvège ont plus de partenaires dans le cadre de leurs coopérations les plus importantes, alors que les coopérations de long terme informelles sont plus communes en Finlande. Les différences structurelles selon l'organisation et l'histoire industrielle sont claires quant à la sous-traitance, celle-ci étant plus présente en Suède qu'en Norvège ou en Finlande. On trouve aussi plusieurs spécificités dans le cas des achats locaux de services d'affaires, mais cette situation peut s'expliquer par les différences dans la présence de services complexes d'affaires selon les localités. À l'exception du conseil en exportation, les exportateurs engagés suédois font plus affaires avec leurs propres services internes. Enfin, on trouve chez les PME finlandaises une plus haute fréquence dans le recours à toutes sortes de services externes pour aider l'exportation. Cela peut être dû au fait que les exportateurs finlandais sont entrés sur les marchés internationaux plus tard que leurs collègues norvégiens et suédois.

Dans le cas des différences régionales, les PME des petites villes sont plus enclines à s'impliquer dans des coopérations occasionnelles de court terme. Cela peut s'expliquer par le fait qu'elles sont plus souvent des 
TABleau 6

Différences entre pays et régions en 1991

des PME exportatrices engagées

\begin{tabular}{|c|c|c|}
\hline & Pays & Région \\
\hline \multicolumn{3}{|l|}{ Ressources internes } \\
\hline $\begin{array}{l}\text { Processus de production } \\
\text { Opinion des entrepreneurs } \\
\text { - niveau technologique }\end{array}$ & $* *$ & \\
\hline $\begin{array}{l}\text { Entrepreneur } \\
\text { - formation commerciale et technique }\end{array}$ & $* *$ & \\
\hline $\begin{array}{l}\text { Employés } \\
\text { - changement dans le niveau de scolarité } \\
\text { - formation (en \% des cadres) } \\
\text { - université ou équivalent }\end{array}$ & $* *$ & $* *$ \\
\hline \multicolumn{3}{|l|}{ Ressources externes } \\
\hline $\begin{array}{l}\text { Relations de coopération } \\
\text { - nombre de partenaires } \\
\text { dans les relations les plus importantes }\end{array}$ & $* *$ & \\
\hline $\begin{array}{l}\text { Formes de coopération } \\
\text { - autres coopérations de long terme } \\
\text { - coopération occasionnelle }\end{array}$ & $*$ & $*$ \\
\hline $\begin{array}{l}\text { Part de sous-traitance dans le chiffre d'affaires } \\
\text { - sous-traitance venant du local } \\
\text { - sous-traitance venant de l'extérieur de la région } \\
\text { - livraison de sous-traitance en dehors de la région }\end{array}$ & $\begin{array}{r}* * \\
* \\
*\end{array}$ & \\
\hline $\begin{array}{l}\text { Services d'affaires (part des achats des PME) } \\
\text { seulement localement }\end{array}$ & & \\
\hline $\begin{array}{l}\text { - transport } \\
\text { - conseil en gestion }\end{array}$ & $*$ & $*$ \\
\hline $\begin{array}{l}\text { - conseil en technologie } \\
\text { - services légaux }\end{array}$ & $* *$ & $* *$ \\
\hline - services publics aux firmes & $* *$ & \\
\hline
\end{tabular}

$* 0,05 ; * * 0,01$.

Différence significative (analyse croisée, $\chi^{2}$, analyse de variance à un seul tour, test $t$ )

Note : Seules les différences significatives des ressources discutées aux tableaux 3 et 4 sont reprises ici.

sous-traitantes. Les différences dans l'achat de services d'affaires reflètent effectivement un niveau d'offre très diversifié : peu de services d'affaires sont disponibles localement dans les milieux ruraux et dans les petites villes.

Les exportateurs suédois engagés voient le climat industriel local autant que le support public local aux industries dans les termes les plus positifs. Au contraire, les entrepreneurs finlandais, en particulier, les évaluent beaucoup plus négativement, même si leur participation à diverses formes de coopération n'est pas nécessairement plus faible. Ce résultat permet peut-être de répondre 
à la question que nous nous posions, à savoir pourquoi les divers répondants ont évalué différemment leur pratique coopérative interfirme.

\section{Conclusion}

On limite généralement le niveau d'internationalisation des PME à leur capacité exportatrice, ce qui a également été fait ici. Pour ce faire, on a divisé ces firmes en trois groupes, soit les PME exportatrices engagées, les exportatrices sporadiques et les non-exportatrices. La part de production exportée et le nombre de pays touchés ont été utilisés comme critères de discrimination. Dans la première partie de notre analyse empirique, nous avons comparé ces trois groupes d'entreprises selon chaque pays nordique quant à leurs ressources internes et leurs possibilités de compléter celles-ci par des ressources externes. En général, les PME exportatrices engagées étaient plus grandes et jouissaient, sur plusieurs aspects, de meilleures ressources internes que les autres PME. Par contre, ces PME-là n'étaient pas tellement différentes des dernières quant à la forme de coopérations verticales ou horizontales. La seule différence importante résidait dans le fait que leurs partenaires provenaient plus souvent de l'étranger.

La deuxième partie de notre analyse concernait les différences nationales et régionales quant aux ressources des PME exportatrices engagées. D'une perspective européenne occidentale, chaque pays nordique et ses régions peuvent être analysés sur deux niveaux opérationnels de l'environnement des PME. Les quatre types de régions (grandes villes, villes industrielles, petites villes et zones rurales) présentent différents systèmes d'organisation hiérarchique et jouent ainsi un rôle clairement défini dans la structure géographique centre / périphérie de chaque pays ${ }^{5}$.

Les données empiriques ont révélé des différences non ambiguës entre les régions et les pays touchant les caractéristiques des exportateurs engagés. Mais les liens entre l'offre de ressources et la localisation des exportateurs engagés n'étaient pas linéaires, et leur structure organisationnelle relative était beaucoup plus complexe. Plus précisément, les différences tant au plan régional que national au regard des ressources internes étaient beaucoup plus faibles que ce que nous avions pu présumer en tenant compte des pressions concurrentielles sur les marchés. Quant aux ressources externes, on a trouvé plus de différences entre les pays qu'entre les régions. Ces différences entre pays pouvaient s'expliquer par les particularités de leurs structures industrielles et

5. Les entrevues incluaient des questions concernant la qualité des services d'affaires locaux, la disponibilité et l'accessibilité d'une main-d'œuvre instruite. Les évaluations des entrepreneurs concordaient avec nos analyses a priori sur la différenciation hiérarchique des milieux locaux et sur l'accès aux ressources. 
aussi par leur performance économique récente. Dans le premier cas, les structures industrielles, l'état de développement de la sous-traitance peut expliquer beaucoup de choses chez les PME, par exemple, l'énergie que les entrepreneurs finlandais ont mis dans la restructuration de cette sous-traitance après la profonde récession qui les a touchés. En général, les profils nationaux des PME demandent des analyses et des descriptions plus fines.

Quant aux qualifications, il est important de souligner que nous avons dû nous concentrer avant tout sur des données quantitatives au sujet des ressources externes; leurs qualifications n'ont pas été prises en compte. Par exemple, il est évident que les relations de coopération peuvent prendre des formes très différentes selon les entrepreneurs et selon les stratégies poutsuivies. Ce problème de qualification touche aussi de façon importante l'interprétation à donner aux résultats ; les résultats trouvés ne nous permettent pas de conclure que les milieux locaux et régionaux sont insignifiants pour les PME. Par exemple, la petite taille et l'éloignement de plusieurs localités peuvent probablement expliquer plusieurs différences dans le niveau d'industrialisation et le fait que l'on trouve peu de nouveaux districts industriels spécialisés dynamiques dans les pays nordiques.

Loin de nous l'idée que nos résultats empiriques puissent régler toute la question du modèle permettant de comprendre le processus d'internationalisation des PME. La base théorique de ce modèle est la division du travail et le développement de réseaux dans lesquels on trouve un nombre important de firmes engagées dans un processus complexe de production. Or, nos résultats nous portent à croire que plus les PME produisent des biens simples, moins elles recourent à plusieurs partenaires. Cela a une influence sur leurs besoins et sur la façon d'acquérir des ressources externes. D'un autre côté, il est aussi clair que l'éventail de produits (produits simples ou complexes) et le rôle joué par la division du travail ne sont pas indépendants l'un de l'autre dans les conditions spatiales de production des pays nordiques.

En particulier, pour les PME qui sont localisées dans des régions périphériques ou dans des petites communautés, elles doivent considérer que l'offre locale de ressources complémentaires sera relativement rare. Cela peut présenter un sérieux problème si les facteurs exogènes tels que l'internationalisation, changent les conditions de concurrence après l'établissement d'une firme spécifique. Les firmes qui, pour diverses raisons, sont situées dans des régions périphériques et dans les petites localités doivent recourir à différentes astuces pour obtenir les ressources stratégiques nécessaires à leur internationalisation. Elles doivent compter davantage sur leurs ressources internes et sur leurs contacts potentiels. Pourtant, cela ne semble pas différent dans le cas des secteurs traditionnels installés en périphérie, comme l'industrie de la transformation du bois qui a développé de bons contacts internationaux. Ces ressources (selon 
chaque pays) peuvent compléter ou même compenser les ressources locales. Dans ce cas, les ressources des milieux locaux et du système industriel national jouent un rôle complémentaire dans l'internationalisation des PME.

De même, les effets interreliés du milieu local sur l'internationalisation des PME ne peuvent être mesurés sur la simple base de données croisées. Aussi, doit-on tenir compte de la position des firmes engagées dans leur système industriel national et de leurs sentiers d'internationalisation. Dans des recherches complémentaires, ce type d'approche qualitative et d'études de cas devrait permettre de compléter notre analyse plutôt quantitative et de faciliter l'élaboration d'un modèle possible de l'internationalisation des PME nordiques.

\section{Remerciements}

Cet article provient d'une étude subventionnée par l'Institut nordique de recherche régionale (NordREFO). En plus des présents auteurs, le groupe de recherche comprenait Poul Rind Christensen (École de commerce danoise du Sud, Kolding, Danemark), Bo Forsström (Institut pour les études européennes, Turku [Åbo], Finlande) et Leif Lindmark (Université d'Umeå, Suède). Les diverses données de l'étude ont été développées par Timo Lautanen (Université de Joensuu, Finlande).

\section{Bibliographie}

ATTIYEH, R.S. et D.L. WENNER (1979), « Critical mass : key to export profits », Business Horizons, décembre.

BIJMOLT, H.A. et P.S. ZWART (1994), « The impact of internal factors on the export success of Dutch small and medium-sized firms », Journal of Small Business Management, vol. 32, $\mathrm{n}^{\circ} 1$, p. 69-83.

BILKEY, W.J. et G. TESAR (1977), «The export behavior of smaller-sized Wisconsin firms », Journal of International Business, printemps-été, p. 93-98.

BLAU, P.M. (1968), «Interaction, social exchange », dans D.I. Sills (éd.), The International Encyclopedia of the Social Sciences, New York, MacMillan.

Boswell, J. (1973), The Rise and Decline of Small Firms, Londres, Allen and Unwin.

BOUWEN, R. et C. STEYAERT (1990), « Construing organizational texture in young entrepreneurial firms », Journal of Management Studies, vol. 27, $\mathrm{n}^{0} 6$, p. 637-649.

Buckley, P.J. et M. CASSON (1985), The Economic Theory of the Multinational Enterprise, New York, MacMillan.

CAVUSGIL, S.T. et J.R. NEVIN (1981), «Internal determinants of export marketing behavior : an empirical investigation », Journal of Marketing Research, vol. 18, $\mathrm{n}^{\circ} 1$, p. 114-119. 
CAVUSGIL, S.T. (1984), "Organizational characteristics associated with export activity », Journal of Management Studies, vol. 21, n 1, p. 3-22.

CHRISTENSEN, P.R. et L. LINDMARK (1993), «In search of regional support in internationalization of small and medium-sized firms - A network perspective ", dans L. Lundqvist et L.O. Persson (éd.), Visions and Strategies in European Integration. A North European Perspective, Berlin, Springer-Verlag, p. 131-151.

Christensen, P.R., H. EsKelinen, B. ForsSTRÖM, L. LindMARK et E. VATNE (1990), «Firms in network: concepts, spatial impacts and policy implications », dans S. Illeris et L. Jackobsen (éd.), Networks and Regional Development, Copenhague, NordREFO / Akademisk Forlag.

CHRISTENSEN, P.R. (1991), «The small and medium-sized exporter's squeeze : empirical evidence and model reflections », Entrepreneurship and Regional Development, vol. 3, p. 49-65.

Czinkota, M.R. et W.J. Johnston (1983), «Exporting : does sales volume make a difference ?», Journal of International Business, printemps-été, p. 147-153.

Diamantopoulos, A. (1988), «Identifying differences between high and lowinvolvement exporters », International Marketing Review, été, p. 56-60.

Dosi, G., K. PAvitT et L. SoEte (1990), «The economics of technical change and international trade », Londres, Harvester Wheatsheaf.

DUNNING, J. (1988), «The eclectic paradigm of international production : a restatement and some possible extensions », Journal of International Business Studies, vol. XIX, no 1 , p. 1-31.

EDMUNDS, S. et K. SARKIS (1986), «Exports : a necessary ingredient in the growth of small business firms ", Journal of Small Business Management, vol. 24, $\mathrm{n}^{\circ} 4$, p. 54-65.

ESKELINEN, H., T. LAUTANEN et B. FORSSTRÖM (1994), « The internationalization of SMEs », dans Four Finnish regions - some tentative observations, dans L. Lundqvist et L.O. Persson (éd.), Northern Perspectives on European Integration, NordREFO, Copenhague, Scandinavian University Press, vol. 1, p. 115-133.

GAROFOLI, G. (1991), «The Italian model of spatial development in the 1970s and 1980 s », dans G. Benko et G.M. Dunford (éd.), Industrial Change and Regional Development, Londres, Belhaven Press.

GRANOVETTER, M.S. (1985), «Economic action and social structure: the problem of embeddedness », American Journal of Sociology, vol. 91, p. 481-510.

GREEN, R. et T. LARSEN (1987), «Environmental shock and export opportunity », International Marketing Review, vol. 4, no 4, p. 30-42.

HARRISON, B. (1992), «Industrial districts : old wine in new bottles? », Regional Studies, vol. $26, n^{0} 5$, p. $469-485$. 
HUll, C.J. et B. HJERN (1987), Helping Small Firms Grow. An Implementation Approach, Londres, Croom Helm.

IMAI, K et Y. BABA (1991), "Systemic innovation and cross border networks: transcending markets and hierarchies to create a new techno-economic system », dans Technology and Productivity, Paris, OCDE, p. 389-407.

JOHANSSON, J. et L.G. MATTSSON (1987), «Interorganizational relations in industrial systems : a network approach compared with the transaction cost approach », Document de travail 1987/7, Department of Business Administration, University of Uppsala.

KAYNAK, E., P.N. GHAURI et T. OlOFSSON-BREDENLÖW (1987), « Export. behavior of small Swedish firms », Journal of Small Business Management, vol. 25, $\mathrm{n}^{\circ} 2$, p. 26-32.

LUNDBERG, L. (1992), «European economic integration and the Nordic countries trade », Journal of Common Market Studies, vol. XXX, $\mathrm{n}^{\circ}$ 2, p. 157-173.

LuNDVALL, B.A. (1988), «Product innovation and user-producer relations», dans G. Dosi et al. (éd.), Technical Change and Economic Theory, Londres, Pinter Publishers.

Marshall, A. (1896), Principles of Economics, $2^{\mathrm{e}}$ édition, Londres, MacMillan.

O'ROURKE, D.A. (1985), « Differences in exporting practices, attitudes, and problems by size of firm », American Journal of Small Business, hiver, p. 68-73.

PEnRose, E.T. (1959), The Theory of the Growth of the Firm, Oxford, Blackwell.

Pfeffer, J. et G.R. SALANCIK (1978), The External Control of Organizations, New York, Harper and Row.

POrTER, M. (1985), Competitive Advantage, New York, Free Press.

RAINNIE, A. (1989), Industrial Relations in Small Firms. Small Isn't Beautiful ?, Londres, Routledge.

REID, S. (1984), «Information acquisition and export entry decisions in small firms », Journal of Business Research, vol. 12, $\mathrm{n}^{\circ} 2$, p. 141-157.

REVE, T. (1990), «The firm as a nexus of internal and external contracts », dans M. Aoki, B. Gustafsson et O.E. Williamson (éd.), The Firm as a Nexus of Treaties, Londres, Sage.

Rothwell, R. et W. Zegveld (1982), Innovation and the Small and Medium Sized Firm, Londres, Frances Pinter.

ScotT, A.J. (1988), Metropolis : From the Division of Labor to Urban Form, Los Angeles, University of California Press. 
SRIRAM, V. et H.J. SAPIENZA (1991), "An empirical investigation of the role of marketing for small exporters », Journal of Small Business Management, vol. 29, $n^{0} 4$, p. 33-43.

SÖlvell, Ö., I. ZANDER et M. Porter (1991), Advantage Sweden, Stockholm, Norstedts.

VATNE, E. (1995), «Local resource mobilization and internationalization strategies in small and medium sized enterprises », Environment and Planning, vol. A 27, $\mathrm{n}^{\circ} 1$, p. 63-80.

WiLliamson, O.E. (1979), « Transaction cost economics : the governance of contractual relations », Journal of Law and Economics, vol. 22, p. 233.

Williamson, O.E. (1985), The Economic Institutions of Capitalism, New York, Free Press. 